\section{A computer program for a randomization test of a difference between independent groups}

\author{
EUGENE S. EDGINGTON, TERRENCE TAERUM, \\ F. PYSH, and ALLAN R. STRAIN \\ University of Calgary, Calgary, Alberta, Canada
}

A program has been developed for a randomization test that serves the purpose of an ordinary t test or one-way analysis of variance without repeated measures. The test determines the probability of getting as large a value of $t$ or $F$ as an obtained value, based on the distribution of $\mathrm{F}$ or $\mathrm{t}$ for all possible ways of dividing the obtained data between the groups being compared. The randomization test has two principal advantages over the $t$ test and analysis of variance: (1) it involves no parametric assumptions, being, in fact, the most powerful of nonparametric tests, and (2) it is a valid test even where there has been no random sampling, provided random assignment has been performed, whereas parametric tests, being based on random-sampling models, are not valid for such applications (Edgington, 1973).

The only serious objection to the widespread employment of randomization tests as alternatives to parametric and rank-order tests is that the large amount of computation required makes such a test impractical. For example, an independent $t$ test with only eight Ss per group, performed as a randomization test, requires (16)!/8! $8 !=12,870$ divisions of the 16 measurements into two groups of 8 measurements, for each of which divisions $t$ (or an equivalent test statistic) must be computed. (An "equivalent test statistic" is one which would provide the same probability value as $t$, although it may be much simpler to compute each time.)

New developments remove this objection. First, high-speed computers can economically perform the thousands of separate computations that may be impractical to perform by a man with pencil and paper or with a desk calculator. And second, where the number of divisions of the data for a randomization test is too large even for a computer to handle economically, an approximate randomization test can be performed by using a random sample of all possible divisions selected by a random number generator routine.

The test statistic used is:

$$
\sum_{j=1}^{m} n_{j} \bar{X}_{\cdot j}^{2}
$$

where $m=$ number of groups, $\bar{X}_{\cdot j}=$ mean of Group $j, n_{j}$ $=$ the number of cases in Group $\mathrm{j}$.

We will now show that this test statistic is an equivalent for the traditional F. F is defined in the usual way by:

$$
F=\frac{S S B / d f B}{S S W / d f W}=\frac{S S B}{S S W} \times \frac{d f W}{d f B} .
$$

In Eq. 2, the ratio dfW/dfB is a constant multiplier and hence does not affect the rank order of the statistic. Since for any possible split of the data, the total sums of squares is a constant, SSB must vary inversely with SSW. As SSB/SSW increases, SSB must necessarily increase. Therefore, the rank order of SSB/SSW will be the same as the rank order of SSB defined in Eq. 3 below (see Bradley, 1968, pp. 80-83).

The test statistic in Eq. 1 may be derived from the deviation sums of squares between groups (SSB) for the equal group size case as:

$$
\operatorname{SSB}=\sum_{j=1}^{m}\left(\bar{X}_{\cdot j}-\bar{X} . .\right)^{2}=\sum_{j=1}^{m} \bar{X}_{\cdot j}^{2}-m \bar{X}_{.}^{2},
$$

where $\overline{\mathrm{X}} \ldots$. = grand mean, and $\mathrm{m}$ and $\overline{\mathrm{X}}_{. \mathrm{j}}$ are defined as in Eq. 1. Because the second term in Eq. 3 is a constant, one need only be interested in comparing the first term for each of the random divisions of the groups. For unequal group sizes, one need only make an adjustment for size of groups as:

$$
\sum_{j=1}^{m} n_{j} \bar{X}_{\cdot j}^{2}
$$

where $m, n_{j}$, and $\bar{X}_{. j}^{2}$ are defined as in Eq. 1 .

In carrying out an exact randomization test, the number of unique combinations for the unequal groups size case (Tu) is given by:

$$
\mathrm{Tu}=\left(\sum_{j=1}^{m} n_{j}\right) ! /\left[\prod_{j=1}^{m}\left(n_{j} !\right)\right],
$$

where $m=$ the number of groups, $n_{j}=$ the number of cases in Group $\mathbf{j}$.

For the equal group size case, the number of unique combinations (Te) is given by:

$$
\mathrm{Te}=\left(\sum_{j=1}^{\mathrm{m}} \mathrm{n}_{\mathrm{j}}\right) ! /\left[\prod_{j=1}^{\mathrm{m}}\left(\mathrm{n}_{\mathrm{j}} !\right) \mathrm{m} !\right] ;
$$

where $m$ and $n_{j}$ are defined as in Eq. 4 .

Limitations. The procedure for generating splits (combinations) is reasonably efficient. Under the exact method, 1,000 splits per second are obtained, while for the approximate procedure the number of combinations 
per second is somewhat slower. The user may opt for an exact solution where the problem configuration does not exceed six groups with any one group not exceeding 30 Ss, and where the number of unique splits is $\leqslant 99,999$. The approximate randomization test procedure will handle up to 10 groups, with a maximum of $300 \mathrm{Ss}$ distributed in each group as a function of the user's problem configuration. For a discussion of the power of the approximate randomization test relative to the exact, see Edgington (1969).

Input. The setup for an analysis is as follows: (1) Title card. (2) Parameter card: Columns 1-5 = the upper limit to the number of splits of the data (maximum of 99,999); Columns 6-7 = the number of independent groups (up to 6 for the exact method and up to 10 for the approximate procedure); Column $8=1$ for $\mathrm{a}$ one-tailed $t$ test and 2 for a two-tailed $t$ or ANOVA $F$; Column $9=1$ for an exact test, otherwise 0 (approximate test). (3) Size of groups card (10F4.0 format). (4) Format card (input format for observations which must describe the data for the largest group in $F$ format). (5) Data deck (observations for each group begin on a new card). (6) Last card (blank card of new title card followed by remainder of program cards as described above).

Output. The computer output for a run includes (a) the means for each group, (b) the value of the statistic given in (1) for the observed data, (c) the traditional $F$ ratio with degrees of freedom, (d) the number of possible splits of the observed data if the exact method is used, and (e) the number of splits in which the statistic given in (1) was greater than or equal to the statistic calculated on the observed data.

Language and Computer. The program is written in FORTRAN and has been implemented on the CDC 6400 computer. It can handle analyses having equal or unequal group sizes and will carry out either an exact or approximate randomization test at the user's discretion. Allowance is also made for carrying out a one-tailed test in the case of two independent groups. The random number generator routine has been adapted from Bulgren (1971) and can be easily modified for machines of different word length than the CDC series.

Availability. A source listing and user's guide which includes sample problems can be obtained free of charge by writing to F. Pysh, University of Calgary, Department of Educational Psychology, Calgary, Alberta, Canada, T2N 1 N4.

\section{REFERENCES}

Bradley, J. V. Distribution-free statistical tests. Englewood Cliffs, N.J: Prentice-Hall, 1968.

Bulgren, $W . A$ computer-assisted approach to elementary statistics: Examples and problems. Belmont, California: Wadsw orth, 1971 .

Edgington, E. S. Approximate randomization tests. Journal of Psychology, 1969, 72, 143-149.

Edgington, E. S. The random-sampling assumption in "Comment on component-randomization tests." Psychological Bulletin, $1973,80,84-85$. 